Discrete Comput Geom 33:143-155 (2005)

DOI: $10.1007 / \mathrm{s} 00454-004-2916-2$

\title{
Covering a Ball with Smaller Equal Balls in $\mathbb{R}^{n}$
}

\author{
Jean-Louis Verger-Gaugry \\ Institut Fourier, University of Grenoble I, \\ UMR5582 (UJF-CNRS), BP 74, \\ 38402 St. Martin d'Hères Cedex, France \\ jlverger@ujf-grenoble.fr
}

\begin{abstract}
We give an explicit upper bound of the minimal number $v_{T, n}$ of balls of radius $\frac{1}{2}$ which form a covering of a ball of radius $T>\frac{1}{2}$ in $\mathbb{R}^{n}, n \geq 2$. The asymptotic estimates of $v_{T, n}$ we deduce when $n$ is large are improved further by recent results of Böröczky, Jr. and Wintsche on the asymptotic estimates of the minimal number of equal balls of $\mathbb{R}^{n}$ covering the sphere $\mathbb{S}^{n-1}$. The optimality of the asymptotic estimates is discussed.
\end{abstract}

\section{Introduction}

Let $T>\frac{1}{2}$ and let $v_{T, n}$ be the minimal number of (closed) balls of radius $\frac{1}{2}$ which can cover a (closed) ball of radius $T$ in $\mathbb{R}^{n}, n \geq 2$. In [R2, pp. 163-164 and Theorem 2] Rogers has obtained the following result:

\section{Theorem 1.1.}

(i) If $n \geq 3$, with $\vartheta_{n}=n \ln n+n \ln (\ln n)+5 n$, we have

$$
1<v_{T, n} \leq \begin{cases}e \vartheta_{n}(2 T)^{n} & \text { if } \quad T \geq \frac{n}{2}, \\ n \vartheta_{n}(2 T)^{n} & \text { if } \quad \frac{n}{2 \ln n} \leq T<\frac{n}{2} .\end{cases}
$$

(ii) If $n \geq 9$ we have

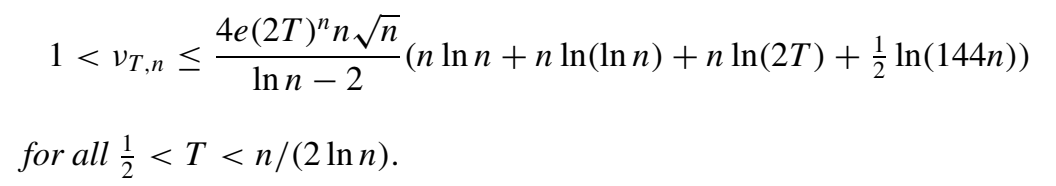


Assertion (i) can easily be extended to the case $n=2$ by invoking [R3, p. 47] so that the strict upper bound $\vartheta_{n}=n \ln n+n \ln (\ln n)+5 n$ of the covering density of equal balls in $\mathbb{R}^{n}$ is still a valid one in this case. Thus the inequalities (1.1) are still true for $n=2$. In the case $n=2$ (see also [K]), on the other hand, the result (ii) does not seem to have been improved since then, see for instance $[\mathrm{GO}],[\mathrm{F}],[\mathrm{S}]$, [R1] or [BL]. This problem is linked to the existence of explicit lower bounds of the packing constant of equal spheres in $\mathbb{R}^{n}[\mathrm{MVG}]$ and to various problems [MR], [IM], [FF], [M].

In this contribution we give an improvement of the upper bound of $\nu_{T, n}$ given by assertion (ii), i.e. when the radius $T$ is less than $n /(2 \ln n)$. Namely, we will prove

Theorem 1.2. Let $n \geq 2$. The following inequalities hold:

(i)

$$
\begin{aligned}
n< & v_{T, n} \leq \frac{7^{4(\ln 7) / 7}}{4} \sqrt{\frac{\pi}{2}} \\
& \times \frac{n \sqrt{n}\left[(n-1) \ln (2 T n)+(n-1) \ln (\ln n)+\frac{1}{2} \ln n+\ln \left(\frac{\pi \sqrt{2 n}}{\sqrt{\pi n}-2}\right)\right]}{T(1-2 / \ln n)(1-2 / \sqrt{\pi n})(\ln n)^{2}} \\
& \times(2 T)^{n} \\
\text { if } \quad 1 & <T<\frac{n}{2 \ln n},
\end{aligned}
$$

(ii)

$$
\begin{aligned}
& n< v_{T, n} \leq \sqrt{\frac{\pi}{2}} \\
& \times \frac{\sqrt{n}\left[(n-1) \ln (2 T n)+(n-1) \ln (\ln n)+\frac{1}{2} \ln n+\ln \left(\frac{\pi \sqrt{2 n}}{\sqrt{\pi n}-2}\right)\right]}{T(1-2 / \ln n)(1-2 / \sqrt{\pi n})} \\
& \times(2 T)^{n} \\
& \text { if } \quad \frac{1}{2}<T \leq 1 .
\end{aligned}
$$

The following question seems fundamental: What are the integers $v_{T, n}$ when $\frac{1}{2}<$ $T, 2 \leq n$ and the corresponding configurations of balls of radius $\frac{1}{2}$ when they form the most economical covering of the closed ball $B(0, T)$ of radius $T$ centred at the origin ?

In Section 3 we recall the recent results of Böröczky, Jr. and Wintsche [BW] on the asymptotic estimates in the sphere covering problem by smaller equal balls when $n$ is large. These estimates allow us to make further improvements on the upper bounds of $v_{T, n}$ (Theorem 3.1), to appreciate the optimality of these upper bounds with respect to known lower bounds and to state some conjectures.

\section{Proof of Theorem 1.2}

The idea of the proof is simple: (i) when $T$ is small enough, it amounts to showing that the sphere $S(0, T)$ can be covered by a collection of $N$ balls of radius $\frac{1}{2}$ suitably 
placed equidistant from the origin, and that this covering to which we add the central ball $B\left(0, \frac{1}{2}\right)$ actually covers the ball $B(0, T)$ itself; in subsection 2 an upper bound of the minimal value of $N$ is calculated from the results given by the lemmas of subsection 1 ; (ii) when $T$ is larger, we proceed recursively using (i) to give an upper bound of $N$. The configuration of balls of radius $\frac{1}{2}$ covering $B(0, T)$ is then ordered by layers, the last layer of balls of radius $\frac{1}{2}$ being at an optimal distance from the origin so as to cover the sphere $S(0, T)$.

1. Caps and Sectors. Let $T>\frac{1}{2}$ and $n \geq 2$ in the following. If the closed ball $B(0, T)$ is covered by $N$ smaller balls of radius $\frac{1}{2}$, the smaller balls will intersect the sphere $S(0, T)$, for a certain proportion of them. The intersection of a closed ball of radius $\frac{1}{2}$ and the sphere $S(0, T)$, if it is not empty, is called a (spherical) cap. To fix the notations we define properly what a cap is and the sector it generates in $B(0, T)$.

Let $h \geq 0$ and let $u$ be a unit vector of $\mathbb{R}^{n}$. We denote by $H_{h, u}$ the affine hyperplane $\left\{z+h u \mid z \in \mathbb{R}^{n}, z \cdot u=0\right\}$ of $\mathbb{R}^{n}$. Assume that $H_{h, u}$ intersects the ball $B(0, T)$, i.e. $h \leq T$. We denote

$$
C_{T, h, u}:=\left\{z \in S(0, T) \mid \frac{z \cdot u}{\|z\|} \geq \frac{h}{T}\right\} .
$$

The $(n-2)$-dimensional sphere $H_{h, u} \cap C_{T, h, u}$ admits $x=\sqrt{T^{2}-h^{2}}$ for the radius. The correspondence between $x \in[0, T]$ and $h \in[0, T]$ is one-to-one. We say that $C_{T, h, u}$ is the cap of chord $2 x$ and of centre $T u$. If a subset $Y$ of $S(0, T)$ is such that there exist $h \geq 0$ and a unit vector $u$ of $\mathbb{R}^{n}$ such that $Y=C_{T, h, u}$, then we say that $Y$ is a cap of chord $2 x$ of $S(0, T)$.

Every cap $C_{T, h, u}$ of chord $2 x$ of $S(0, T)$ generates a sector in $B(0, T)$. We denote it by

$$
\mathscr{S}(T, h, u):=\left\{z \in B(0, T) \mid \frac{z \cdot u}{\|z\|} \geq \frac{h}{T}\right\} .
$$

We denote by $V_{(T, x)}$ (indexing with $x$ instead of $h$ ) the volume of a sector generated by a cap of chord $2 x$ in $S(0, T)$ with $x \leq T$. Let $\omega_{n}:=\pi^{n / 2} / \Gamma(1+n / 2)$ so that the ( $n$-dimensional) volume of a ball of radius $T$ in $\mathbb{R}^{n}$ is $\omega_{n} T^{n}$.

Lemma 2.1. We have

$$
\frac{\omega_{n-1}}{\omega_{n}} \geq \frac{1}{\sqrt{2 \pi}} \sqrt{n}\left(1-\frac{2}{\sqrt{\pi n}}\right) .
$$

Proof. The following inequalities are classical [V, p. 171]:

$$
\frac{\omega_{n-1}}{\omega_{n}} \geq \begin{cases}\frac{1}{\sqrt{2 \pi}} \sqrt{n}\left(1+\left(\frac{n}{2 e}\right)^{n / 2} \frac{1}{(n / 2) !}\right)^{-1} & \text { if } n \text { is even } \\ \frac{1}{\sqrt{2 \pi}} \sqrt{n+1}\left(1-\left(\frac{n+1}{2 e}\right)^{(n+1) / 2} \frac{1}{((n+1) / 2) !}\right) & \text { if } n \text { is odd. }\end{cases}
$$

By Stirling's formula we deduce the result. 
Lemma 2.2. Let $0<x<T$. Let $n$ be odd and put $\gamma=(n-1) / 2$. The volume $V_{(T, x)}$ of a sector in $B(0, T) \subset \mathbb{R}^{n}$ generated by a cap of chord $2 x$ in $S(0, T)$ is equal to

$$
\begin{aligned}
\omega_{n-1} x^{n-1}[ & \frac{\sqrt{T^{2}-x^{2}}}{n}+\frac{2\left(T-\sqrt{T^{2}-x^{2}}\right)}{n+1} \sum_{j=0}^{\gamma} \frac{\gamma !(\gamma+1) !}{(\gamma+1+j) !(\gamma-j) !} \\
& \left.\times\left(\frac{T-\sqrt{T^{2}-x^{2}}}{T+\sqrt{T^{2}-x^{2}}}\right)^{j}\right] .
\end{aligned}
$$

It satisfies the relations

(i)

$$
V_{(T, x)}=x^{n} V_{(T / x, 1)},
$$

(ii)

$$
\frac{T}{n x} \leq \frac{2 n(T / x)+(1-n) \sqrt{(T / x)^{2}-1}}{n(n+1)} \leq \frac{1}{\omega_{n-1}} V_{(T / x, 1)} .
$$

Proof. Let us show (2.3). The first term $\omega_{n-1} x^{n-1}\left(\sqrt{T^{2}-x^{2}} / n\right)$ is the volume of the truncated cone $\{z \in \mathscr{S}(T, h, u) \mid z \cdot u \leq h\}$ with $h=\sqrt{T^{2}-x^{2}}$. The second term in (2.3) is the volume of $\{z \in \mathscr{S}(T, h, u) \mid z \cdot u \geq h\}$ : any point of $C_{T, \sqrt{T^{2}-x^{2}}, u}$ which is at distance $t$ from $H_{\sqrt{T^{2}-x^{2}}, u}$ is at distance $\left(x^{2}-t^{2}-2 t \sqrt{T^{2}-x^{2}}\right)^{1 / 2}$ from the line $\mathbb{R} u$. Hence, this volume equals

$$
\int_{0}^{T-\sqrt{T^{2}-x^{2}}} \omega_{n-1}\left[x^{2}-t^{2}-2 t \sqrt{T^{2}-x^{2}}\right]^{(n-1) / 2} d t .
$$

It is obtained by integration by parts, $\gamma$ times, of the integral

$$
\omega_{n-1} \int_{0}^{\alpha}(\alpha-t)^{\gamma}(t-\beta)^{\gamma} d t
$$

with $\alpha=T-\sqrt{T^{2}-x^{2}}$ and $\beta=-T-\sqrt{T^{2}-x^{2}}$.

Relation (2.4) is obvious. Let us show (2.5). We deduce it from the fact that the summation in (2.3) has positive terms and is greater than its first term which is 1.

Lemma 2.3. Assume $n \geq 2$ even and $0<x<1$. The volume $V_{(T, x)}$ of a sector in $B(0, T) \subset \mathbb{R}^{n}$ generated by a cap of chord $2 x$ in $S(0, T)$ satisfies the relations:

(i)

$$
V_{(T, x)}=x^{n} V_{(T / x, 1)},
$$

(ii)

$$
\frac{T}{n x} \leq \frac{2 n(T / x)+(2-n) \sqrt{(T / x)^{2}-1}}{n(n+2)} \leq \frac{1}{\omega_{n-1}} V_{(T / x, 1)}
$$


Proof. Equality (2.7) is obvious. In order to prove (2.8), observe that the function $t \rightarrow(\alpha-t)(t-\beta)$ defined on the interval $[0, \alpha]$ is valued in the interval $[0,1]$ since it lies below the horizontal line of $y$-coordinate $-\alpha \beta=x^{2}<1$. We deduce the following inequalities:

$$
(\alpha-t)^{(n+1) / 2}(t-\beta)^{(n+1) / 2} \leq(\alpha-t)^{n / 2}(t-\beta)^{n / 2} \leq(\alpha-t)^{(n-1) / 2}(t-\beta)^{(n-1) / 2}
$$

for all $t \in[0, \alpha]$. From (2.6) in the proof of Lemma 2.2 we deduce a lower bound of the volume of the convex hull of $C_{T, \sqrt{T^{2}-x^{2}}, u}$ for $n$ even using the preceding $n$ odd case of Lemma 2.2: changing $n$ to $n+1$ now odd in the computation of the lower bound of the summation in (2.3). Note that the computation of the volume of $\{z \in \mathscr{S}(T, h, u) \mid z \cdot u \leq$ $h$ \} with $h=\sqrt{T^{2}-x^{2}}$ still gives $\omega_{n-1} x^{n-1}\left(\sqrt{T^{2}-x^{2}} / n\right)$ for $n$ even so that the first term of $V_{(T, x)}$ remains the same as in the $n$ odd case. We deduce inequality (2.8).

Lemma 2.4. Let $0<x \leq \frac{1}{2}$. Let $D$ be a point of the cap $C_{T, \sqrt{T^{2}-1 / 4}, u} \subset S(0, T) \subset \mathbb{R}^{n}$ at a distance $x$ from the line $\mathbb{R} u$. Let $B$ denote the unique point which lies in the intersection of $C_{T, \sqrt{T^{2}-1 / 4}, u} \cap H_{\sqrt{T^{2}-1 / 4}, u}$ with the plane $(0, D, T u)$ with the property that it is the closest to $D$. If $\eta$ denotes the distance between $D$ and the line $O B$, we have the following relation between $x, T$ and $\eta$ :

$$
\begin{aligned}
& x=\frac{1}{2} \sqrt{1-\left(\frac{\eta}{T}\right)^{2}}-\frac{\eta}{2} \sqrt{4-\frac{1}{T^{2}}}, \quad \text { equivalently } \\
& \eta=\frac{1}{2} \sqrt{1-\left(\frac{x}{T}\right)^{2}}-\frac{x}{2} \sqrt{4-\frac{1}{T^{2}}}
\end{aligned}
$$

Proof. Let $\psi$ be the angle between lines $O B$ and $O D$, and let $\psi^{\prime}$ be the angle between lines $O D$ and $\mathbb{R} u$, so that $\sin (\psi)=\eta / T$ and $\sin \left(\psi^{\prime}\right)=x / T$. Since $\sin \left(\psi+\psi^{\prime}\right)=1 / 2 T$ we obtain

$$
1=2 x \sqrt{1-(\eta / T)^{2}}+2 \eta \sqrt{1-(x / T)^{2}} .
$$

This expression is symmetrical in $x$ and $\eta$. It is now easy to deduce, from it, the expression of $x$ as a function of $\eta$, as stated by (2.9).

Lemma 2.5. Let $u$ s assume that a collection of $N$ balls $\left(B\left(c_{j}, \frac{1}{2}\right)\right)_{j=1,2, \ldots, N}$ of $\mathbb{R}^{n}$ is such that (i) for all $j=1,2, \ldots, N, B\left(c_{j}, \frac{1}{2}\right) \cap S(0, T)$ is a cap of chord 1 in $S(0, T)$ and (ii) these $N$ caps form a covering of $S(0, T)$. Then (i) if $T>\sqrt{2} / 2$, the union

$$
\bigcup_{j=1}^{N} B\left(c_{j}, \frac{1}{2}\right) \quad \text { covers the annulus } \quad\left\{z \in \mathbb{R}^{n} \mid T-\frac{1}{2 T} \leq\|z\| \leq T\right\}
$$

of the ball $B(0, T)$; (ii) if $\frac{1}{2}<T \leq \sqrt{2} / 2$ this union covers $B(0, T)$.

Proof. Any such ball $B\left(c_{j}, \frac{1}{2}\right)$ covers the part of the sector

$$
\left\{z \in \mathscr{S}\left(T, \sqrt{T^{2}-\frac{1}{4}}, O c_{j} /\left\|O c_{j}\right\|\right) \mid \alpha T \leq\|z\|\right\}
$$


with $\alpha$ to be determined. To compute $\alpha$, we consider two adjacent balls, say $B\left(c_{1}, \frac{1}{2}\right)$ and $B\left(c_{2}, \frac{1}{2}\right)$, such that the intersection of the respective caps $B\left(c_{1}, \frac{1}{2}\right) \cap S(0, T)$ and $B\left(c_{2}, \frac{1}{2}\right) \cap S(0, T)$ is reduced to one point. Then, on the line $\mathrm{O}\left(\left(c_{1}+c_{2}\right) / 2\right)$, it is easy to check that all points $z$ such that $T-1 / 2 T \leq\|z\| \leq T$ are covered. This gives $\alpha=1-1 / 2 T^{2}$. Now, since the caps $B\left(c_{j}, \frac{1}{2}\right) \cap S(0, T)$ form a covering of $S(0, T)$, the balls $B\left(c_{j}, \frac{1}{2}\right)$ form a covering of the annulus $\left\{z \in \mathbb{R}^{n} \mid \alpha T \leq\|z\| \leq T\right\}$. The last assertion is obvious.

Let us consider $N(\geq 1)$ distinct points $M_{1}, M_{2}, \ldots, M_{N}$ of $S(0, T) \subset \mathbb{R}^{n}$. We consider that they are the respective centres of caps of chord $2 x$ of $S(0, T)$. We denote by $\theta_{(T, x)}\left(M_{1}, M_{2}, \ldots, M_{N}\right)$ the proportion of $S(0, T)$ occupied by these caps. In other terms, with $u_{i}:=O M_{i} /\left\|O M_{i}\right\|$ for all $i=1,2, \ldots, N$, we have

$$
\theta_{(T, x)}\left(M_{1}, M_{2}, \ldots, M_{N}\right):=\frac{\operatorname{Vol}_{n-1}\left(\bigcup_{i=1}^{N} C_{T, \sqrt{T^{2}-x^{2}, u_{i}}}\right)}{\operatorname{Vol}_{n-1}(S(0, T))}
$$

Lemma 2.6. Let $N \geq 1$ and $x \in\left(0, \frac{1}{2}\right]$. The mean $E \theta(N, T, x)$ of $\theta_{(T, x)}\left(M_{1}, M_{2}, \ldots\right.$, $\left.M_{N}\right)$ over all possibilites of collections of $N$ distinct points $\left(M_{1}, M_{2}, \ldots, M_{N}\right)$ of $S(0, T)$ is equal to

$$
E \theta(N, T, x)=1-\left(1-\frac{V_{(T, x)}}{\omega_{n} T^{n}}\right)^{N}
$$

Proof. Let $M_{1}, M_{2}, \ldots, M_{N}$ be $N$ points of $S(0, T)$. We define

$$
p_{i}=\frac{\operatorname{Vol}_{n-1}\left(C_{T, \sqrt{T^{2}-x^{2}}, u_{i}}\right)}{\operatorname{Vol}_{n-1}(S(0, T))}, \quad i=1,2, \ldots, N,
$$

the probability that a point $M \in S(0, T)$ belongs to the cap of chord $2 x$ of centre $M_{i}$. It is the probability, hence independent of $i$, that $M_{i}$ belongs to the cap of chord $2 x$ of centre $M$. We have $p_{i}=V_{(T, x)} / \omega_{n} T^{n}$. Therefore, the probability that $M$ belongs to none of the caps of chord $2 x$ of centre $M_{i}$ for all $i=1,2, \ldots, N$ is, by the independence of the points, the product of the probabilities that none of the $M_{i}$ 's belongs to the cap of chord $2 x$ of centre $M$, that is the product

$$
\left(1-\frac{V_{(T, x)}}{\omega_{n} T^{n}}\right)^{N}
$$

This value is independent of the collection of points $\left\{M_{i}\right\}$. We deduce the mean $E \theta(N, T$, $x$ ) by complementarity.

\section{Proof of Theorem 1.2}

Proposition 2.7. Let $0<x<\frac{1}{2}$. With $\eta(x)=\frac{1}{2} \sqrt{1-(x / T)^{2}}-(x / 2) \sqrt{4-1 / T^{2}}$, if

$$
N \geq \frac{\omega_{n} T^{n}}{V_{(T, x)}} \ln \left(\frac{\omega_{n} T^{n}}{V_{(T, \eta(x))}}\right),
$$


then there exists a collection of $N$ distinct caps of centres $M_{1}, M_{2}, \ldots, M_{N}$ of chord 1 of $S(0, T) \subset \mathbb{R}^{n}$ satisfying

$$
\ln \left(\frac{1}{1-\theta_{(T, x)}\left(M_{1}, M_{2}, \ldots, M_{N}\right)}\right)>N \frac{V_{(T, x)}}{\omega_{n} T^{n}},
$$

which covers $S(0, T)$.

Proof. Given $x \in\left(0, \frac{1}{2}\right]$ there exists at least one collection of caps $\left\{C_{T, \sqrt{T^{2}-x^{2}}, u_{i}} \mid i=\right.$ $1,2, \ldots, N\}$ of centres $M_{1}, M_{2}, \ldots, M_{N}$, where the unit vectors $u_{i}:=O M_{i} /\left\|O M_{i}\right\|$ are all distinct, such that relation (2.11) is true since, after Lemma 2.6, the mean $E \theta(N, T, x)$ is equal to $1-\left(1-V_{(T, x)} / \omega_{n} T^{n}\right)^{N}$ and that

$$
\ln \left(\frac{1}{1-E \theta(N, T, x)}\right)=-N \ln \left(1-\frac{V_{(T, x)}}{\omega_{n} T^{n}}\right)>N \frac{V_{(T, x)}}{\omega_{n} T^{n}} .
$$

Note that the points $M_{1}, M_{2}, \ldots, M_{n}$ depend upon $x$. Keeping the centres $M_{1}, M_{2}$, $\ldots, M_{N}$ fixed and putting caps of chord 1 instead of $2 x$ around them, we obtain a new collection of caps. Let us show that this new collection of caps of chords 1 of $S(0, T)$ forms a covering. We assume that it does not and will show the contradiction.

Then there exists a point $M \in S(0, T)$ such that

$$
M \notin \bigcup_{i=1}^{N} C_{T, \sqrt{T^{2}-1 / 4}, u_{i}} .
$$

We write $u:=O M /\|O M\|$ for the unit vector on the line $O M$. At worse, $M$ lies close to the boundary of the domain $\bigcup_{i=1}^{N} C_{T, \sqrt{T^{2}-1 / 4}, u_{i}}$, hence close to the boundary of one of the caps $C_{T, \sqrt{T^{2}-1 / 4}, u_{i}}$ of chord 1. We can now apply Lemma 2.4 as if $M$ were on this boundary: $\eta=\eta(x)$ is strictly positive since $x<\frac{1}{2}$ by (2.9). Therefore the cap $C_{T, \sqrt{T^{2}-\eta(x)^{2}}, u}$ is not trivial and is disjoint from the union

$$
\bigcup_{i=1}^{N} C_{T, \sqrt{T^{2}-x^{2}}, u_{i}} .
$$

This means that

$$
1-\theta_{(T, x)}\left(M_{1}, M_{2}, \ldots, M_{N}\right)>\theta_{(T, \eta(x))}(M)>0 .
$$

Therefore

$$
\ln \left(\frac{1}{1-\theta_{(T, x)}\left(M_{1}, M_{2}, \ldots, M_{N}\right)}\right)<\ln \left(\frac{1}{\theta_{(T, \eta(x))}(M)}\right) .
$$

From (2.12) we deduce the relation

$$
N \frac{V_{(T, x)}}{\omega_{n} T^{n}}<\ln \left(\frac{\omega_{n} T^{n}}{V_{(T, \eta(x))}}\right) .
$$

Hence the contradiction. 
By Lemma 2.1 and (2.4), (2.5), (2.7), and (2.8), we deduce

$$
\begin{aligned}
\frac{\omega_{n} T^{n}}{V_{(T, x)}} \ln \left(\frac{\omega_{n} T^{n}}{V_{(T, \eta(x))}}\right)= & \frac{\omega_{n}}{\omega_{n-1}} \frac{n T^{n}}{x^{n}} \frac{\omega_{n-1}}{n V_{(T / x, 1)}} \ln \left(\frac{\omega_{n}}{\omega_{n-1}} \frac{n T^{n}}{(\eta(x))^{n}} \frac{\omega_{n-1}}{n V_{(T /(\eta(x)), 1)}}\right) \\
\leq & \sqrt{\frac{\pi}{2}} \frac{\sqrt{n}(2 T)^{n}(1-4 \eta(x))^{-n / 2}}{T(1-2 / \sqrt{\pi n})} \\
& \times\left[-(n-1) \ln (\eta(x))+(n-1) \ln T+\ln \left(\frac{\sqrt{2} \pi n}{\sqrt{\pi n}-2}\right)\right] .
\end{aligned}
$$

In Proposition 2.7 we can take any $x$, hence any $\eta$, in the open interval $\left(0, \frac{1}{2}\right)$ such that condition (2.11) is satisfied. We chose $\eta$ and $x=x(\eta)$ as functions of $n$ only with $\eta$ tending monotonically to zero when $n$ goes to infinity, hence $x$ tending to $\frac{1}{2}$. This gives a minimal integer

$$
\left\lfloor\frac{\omega_{n} T^{n}}{V_{(T, x)}} \ln \left(\frac{\omega_{n} T^{n}}{V_{(T, \eta(x))}}\right)\right\rfloor+1
$$

for obtaining the covering property of $S(0, T)$ as a function of $n$ and $T$ only.

We now state the central problem $(\mathrm{P})$.

(P) The problem consists now in finding, in the set of strictly positive monotone decreasing functions $f(x)$ defined on $\left(\frac{1}{4},+\infty\right)$ such that $\lim _{x \rightarrow+\infty} f(x)=0$, one function for which $-(1-4 f(x))^{-x / 2} \ln (f(x))$ goes the slowest to $+\infty$ when $x$ tends to $+\infty$.

We do not solve this problem here. We simply take $f(x)=1 /(2 x u(x))$ with $u(x)$ an increasing monotone continuous function such that $\lim _{x \rightarrow+\infty} u(x)=+\infty$, in particular, $u(x)=\ln x$. By reporting this function in (2.13) we take $\eta=1 /(2 n \ln n), n \geq 3$. This gives an expression of $x$ as a function of $n$ from (2.9). This function represents a fairly good compromise.

The second member of inequality (2.10) appears as a configurational entropy which has to be exceeded for the existence of a certain configuration (at least one) of equal caps of chord 1 for covering $S(0, T)$. However, condition (2.11) is non-constructive.

We now make explicit the second member of inequality (2.13) with $\eta=1 /(2 n \ln n)$. Thus, for all $n \geq 2$, since $(1-2 /(n \ln n))^{-n / 2}<(1-2 /(\ln n))^{-1}$, we obtain

$$
\begin{aligned}
& \sqrt{\frac{\pi}{2}} \frac{\sqrt{n}}{T}\left(\frac{(2 T)^{n}}{(1-2 / \ln n)(1-2 / \sqrt{\pi n})}\right) \\
& \quad \times\left[(n-1) \ln (2 T n \ln n)+\frac{1}{2} \ln n+\ln \left(\frac{\pi \sqrt{2 n}}{\sqrt{\pi n}-2}\right)\right]
\end{aligned}
$$

for $\frac{1}{2}<T \leq 1$.

By Lemma 2.5 , if $\frac{1}{2}<T \leq 1$, then, in order to cover the ball $B(0, T)$ by balls of radius $\frac{1}{2}$, it suffices to put a ball of radius $\frac{1}{2}$ centred at the origin (not necessary if $\frac{1}{2}<T \leq \sqrt{2} / 2$ ) and to put a collection of $N$ balls (with $N$ chosen minimal) given by Proposition 2.7 around such that their intersections with $S(0, T)$ are caps of chord 1 
which cover $S(0, T)$. This total number of balls, $N+1$, is certainly exceeded by (2.14). This proves assertion (i) in Theorem 1.2.

Let us prove assertion (ii) in Theorem 1.2. If $T>1$, we proceed inductively using Lemma 2.5. We cover $B(0, T)$ as follows. We put a ball of radius $\frac{1}{2}$ centred at the origin. Then we put balls of radius $\frac{1}{2}$ in such a way that their intersections with the spheres $S\left(0, T_{m}\right)$ are caps of chord 1 which cover $S\left(0, T_{m}\right)$, where the decreasing sequence $\left\{T_{m}\right\}$ is defined by $T_{0}=T, T_{1}=T_{0}-1 / 2 T_{0}, \ldots, T_{m}=T_{m-1}-1 / 2 T_{m-1}, \ldots$ with $m \in\left\{0,1, \ldots, m_{0}\right\}$ and $m_{0}$ defined by the condition that $T_{m_{0}} \leq 1$ and $T_{m_{0}-1}>1$. Since, for all integers $m \in\left\{0,1, \ldots, m_{0}\right\}$, we have

$$
T-\frac{m}{2 T} \geq T_{m}
$$

the total number of balls of radius $\frac{1}{2}$ disposed in such a configuration required for covering $B(0, T)$ is certainly less than

$$
\begin{aligned}
\sum_{m=0}^{m_{0}} & \left(2\left(T-\frac{m}{2 T}\right)\right)^{n} \frac{\sqrt{\pi n}(1-2 / \ln n)^{-1}}{T \sqrt{2}(1-2 / \sqrt{\pi n})} \\
& \times\left[(n-1) \ln \left(2\left(T-\frac{m}{2 T}\right) n \ln n\right)+\frac{\ln n}{2}+\ln \left(\frac{\pi \sqrt{2 n}}{\sqrt{\pi n}-2}\right)\right] \\
\leq & \frac{\sqrt{\pi n}(1-2 / \ln n)^{-1}}{T \sqrt{2}(1-2 / \sqrt{\pi n})}\left[(n-1) \ln (2 T n \ln n)+\frac{\ln n}{2}+\ln \left(\frac{\pi \sqrt{2 n}}{\sqrt{\pi n}-2}\right)\right] \\
& \times \sum_{m=0}^{m_{0}}\left(2\left(T-\frac{m}{2 T}\right)\right)^{n} .
\end{aligned}
$$

However,

$$
\sum_{m=0}^{m_{0}}\left(2\left(T-\frac{m}{2 T}\right)\right)^{n} \leq(2 T)^{n} \sum_{m=0}^{m_{0}} e^{-n m / T^{2}} \leq(2 T)^{n} \sum_{m=0}^{+\infty} e^{-n m / T^{2}}=\frac{(2 T)^{n}}{1-e^{-n / T^{2}}} .
$$

Since $T<n /(2 \ln n)$, we have

$$
\frac{e^{n / T^{2}}}{e^{n / T^{2}}-1}<\frac{e^{4(\ln n)^{2} / n}}{e^{4(\ln n)^{2} / n}-1}<\frac{n}{4(\ln n)^{2}} e^{4(\ln n)^{2} / n} .
$$

The function $t \rightarrow(\ln t)^{2} / t$ reaches its maximum on $[2,+\infty)$ at $t=e^{2}$. Hence, for all integers $n \geq 2$, we have $(\ln n)^{2} / n \leq(\ln 7)^{2} / 7$. We deduce that

$$
\sum_{m=0}^{m_{0}}\left(2\left(T-\frac{m}{2 T}\right)\right)^{n} \leq \frac{e^{4(\ln 7)^{2} / 7}}{4} \frac{n(2 T)^{n}}{(\ln n)^{2}}
$$

with a constant $e^{4(\ln 7)^{2} / 7} / 4=2.176 \ldots$ This gives assertion (ii).

As for the strict lower bound $n$ in (2.3) and (2.4), it obviously comes from the dimension of the ambiant space: $n$ balls being placed along the $n$ coordinates axis of any basis of $\mathbb{R}^{n}$ never cover $B(0, T)$ when $T>\frac{1}{2}$. 


\section{Asymptotic Estimates: Results and Conjectures}

Rogers [R2] constructed certain economic coverings of a larger Euclidean ball by equal smaller balls. When $T$ is large $(T>n / 2)$ he has computed an upper bound on the quantity $v_{T, n}$ that is close to being optimal up to a $\ln n$ factor. On the other hand, his upper bounds are of higher order when $T<n / 2$, and it is the object of Theorem 1.2 to improve them in the case $T<n / 2 \ln (n)$.

In this section we reformulate Theorem 1.2 in terms of asymptotic estimates. Then we state further improved upper bounds on the quantity $v_{T, n}$ for $T<n / 2$ that are most probably close to being optimal up to a $\ln n$ factor. In addition lower bounds on the quantity $v_{T, n}$ are discussed. The arguments for further improvements use recent results of $[\mathrm{BW}]$.

What follows only discusses the order of the bounds, hence we introduce corresponding notation: given non-negative functions $f$ and $g$, if $f(n)<c \cdot g(n)$ for a positive absolute constant $c$, then we write $f(n) \ll g(n)$, or $g(n) \gg f(n)$, or $f(n)=O(g(n))$.

The starting point is the following list of estimates by Rogers [R2]:

$$
\begin{array}{ll}
v_{T, n} \ll n \ln n \cdot(2 T)^{n} & \text { if } \quad T \geq \frac{n}{2} ; \\
v_{T, n} \ll n^{2} \ln n \cdot(2 T)^{n} & \text { if } \quad \frac{n}{2 \ln n} \leq T<\frac{n}{2} ; \\
v_{T, n} \ll n^{2} \sqrt{n} \cdot(2 T)^{n} & \text { if } \quad \frac{1}{2}<T<\frac{n}{2 \ln n} .
\end{array}
$$

Most probably (3.1) cannot be improved with the present methods, and it is actually optimal up to a $\ln n$ factor (see (3.10)). Theorem 1.2 improves (3.3) into the following estimates:

$$
\begin{aligned}
v_{T, n} \ll \frac{n^{2} \sqrt{n}}{T \ln n} \cdot(2 T)^{n} \quad \text { if } 1<T \leq \frac{n}{2 \ln n} \quad \text { where } \quad \frac{n^{2} \sqrt{n}}{T \ln n}>n \sqrt{n} ; \\
v_{T, n} \ll n \sqrt{n} \ln n \cdot(2 T)^{n-1} \quad \text { if } \quad \frac{1}{2}<T \leq 1 .
\end{aligned}
$$

Using some bounds of [BW, subsection 3.2] estimates (3.2) and (3.3) of Rogers and the present estimates (3.4) and (3.5) can be further improved as follows.

Theorem 3.1. The following asymptotic estimates hold:

$$
\begin{aligned}
v_{T, n} & \ll n \ln n \cdot(2 T)^{n} \quad \text { if } T \geq \frac{\sqrt{n}}{2} ; \\
v_{T, n} & \ll \frac{n \sqrt{n} \ln n}{T} \cdot(2 T)^{n} \quad \text { if } \quad 1 \leq T \leq \frac{\sqrt{n}}{2} ; \\
\frac{v_{T, n}}{(2 T)^{n-1}} & \ll n \sqrt{n} \cdot \sqrt{T-\frac{1}{2}} \cdot \ln 8\left(T-\frac{1}{2}\right) n \quad \text { if } \frac{1}{2}+\frac{1}{4 n} \leq T \leq 1 ; \\
v_{T, n} & <2 n \quad \text { if } \quad \frac{1}{2}<T \leq \frac{1}{2}+\frac{1}{4 n} .
\end{aligned}
$$

Observe that the estimates in the list change continuously as $T$ increases (up to absolute constant factors). 
In order to provide a feeling about the optimality of the estimates above, we list the corresponding known and conjectured lower bounds:

$$
\begin{array}{cl}
v_{T, n} \gg n \cdot(2 T)^{n} & \text { if } \quad T \geq \frac{n}{2} \quad \text { or } T=\frac{\sqrt{n}}{2} ; \\
v_{T, n} \gg \frac{n \sqrt{n}}{T} \cdot(2 T)^{n} & \text { if } \quad 1 \leq T \leq \frac{\sqrt{n}}{2}, \quad \text { conjectured; } \\
\frac{v_{T, n}}{(2 T)^{n}} \gg n \sqrt{n} \cdot \sqrt{T-\frac{1}{2}} \quad \text { if } \quad \frac{1}{2}+\frac{1}{4 n} \leq T \leq 1, \quad \text { conjectured; } \\
v_{T, n}>n \quad \text { if } \quad \frac{1}{2}<T \leq \frac{1}{2}+\frac{1}{4 n} .
\end{array}
$$

It is conjectured [BW] that (3.10) holds for any $T \geq \sqrt{n} / 2$ but Böröczkr, Jr. and Wintsche only verified that

$$
v_{T, n} \gg \frac{n \sqrt{n}}{T} \cdot(2 T)^{n} \quad \text { if } \quad \frac{\sqrt{n}}{2} \leq T \leq \frac{n}{2} .
$$

The quantity $v_{T, n} /(2 T)^{n}$ is the minimal density of a covering of a ball of radius $T$ by balls of radius $\frac{1}{2}$. Since $\nu_{T, n}$ balls of radius $\frac{1}{2}$ cover the ball of radius $T$, readily

$$
v_{T, n}>(2 T)^{n} .
$$

\subsection{Covering a Sphere}

The arguments for (3.6)-(3.14) depend on estimates on the minimal number of equal balls covering a sphere. Let $\tilde{v}_{n}(T, \varrho)$ denote the minimal number of balls of radius $\varrho$ in $\mathbb{R}^{n}$ that cover the sphere $S(0, T)$ of radius $T$. The number $\tilde{v}_{n}(T, \varrho)$ corresponds to an optimal function in problem (P) (in Section 2). A better upper estimate of $\tilde{v}_{n}(T, \varrho)$ is given by Corollary 1.2 of [BW]:

$$
\begin{aligned}
& \tilde{v}_{n}(T, \varrho) \ll n \sqrt{n} \ln n \cdot\left(\frac{T}{\varrho}\right)^{n-1} \quad \text { if } \quad T \geq 2 \varrho ; \\
& \frac{\tilde{v}_{n}\left(T, \frac{1}{2}\right)}{(2 T)^{n-1}} \ll n \sqrt{n} \cdot \sqrt{T-\frac{1}{2}} \cdot \ln 8\left(T-\frac{1}{2}\right) n \quad \text { if } \quad \frac{1}{2}+\frac{1}{4 n} \leq T \leq 1 .
\end{aligned}
$$

Concerning lower bounds, Example 6.3 of [BW] says

$$
\tilde{v}_{n}\left(T, \frac{1}{2}\right) \gg n \sqrt{n} \cdot(2 T)^{n-1} \quad \text { if } \quad T \geq \frac{\sqrt{n}}{2} .
$$

It is conjectured [BW] that

$$
\begin{aligned}
& \tilde{v}_{n}\left(T, \frac{1}{2}\right) \gg n \sqrt{n} \cdot(2 T)^{n-1} \quad \text { if } \quad 1 \leq T<\frac{\sqrt{n}}{2} ; \\
& \frac{\tilde{v}_{n}\left(T, \frac{1}{2}\right)}{(2 T)^{n-1} \gg n \sqrt{n} \cdot \sqrt{T-\frac{1}{2}}} \quad \text { if } \quad \frac{1}{2}+\frac{1}{4 n} \leq T \leq 1 .
\end{aligned}
$$




\subsection{Proofs of the Improved Upper Bounds in Theorem 3.1}

If $T \geq \sqrt{n} / 2$, then let $\varrho=(1-1 / n) \frac{1}{2}$. Given any $R \geq \frac{1}{2}$, we cover $S(0, R)$ with $\tilde{v}_{n}(T, \varrho)$ balls of radius $\varrho$ in a way that each ball intersects $S(0, R)$ in an $(n-2)$-sphere of radius $\varrho$. Since $\sqrt{\frac{1}{4}-\varrho^{2}}>1 / 2 \sqrt{n}$, balls of the same centre and of radius $\frac{1}{2}$ cover the annulus between $S(0, R)$ and $S(0, R-1 / 2 \sqrt{n})$. Writing $m$ to denote the maximal integer such that $T-m / 2 \sqrt{n} \geq \frac{1}{2}$, it follows by (3.15) that

$$
\begin{aligned}
v_{T, n} & \leq 1+\sum_{i=0}^{m} \tilde{v}_{n}\left(T-\frac{i}{2 \sqrt{n}}, \varrho\right) \\
& \ll \varrho^{-(n-1)} n \sqrt{n} \ln n \sum_{i=0}^{m}\left(T-\frac{i}{2 \sqrt{n}}\right)^{n-1} \\
& \ll 2^{n-1} n \sqrt{n} \ln n \cdot 2 \sqrt{n} \int_{0}^{T+1 / 2 \sqrt{n}} x^{n-1} d x \\
& \ll n \ln n \cdot(2 T)^{n} .
\end{aligned}
$$

If $1 \leq T \leq \sqrt{n} / 2$, then the argument is based on Lemma 2.5 , which actually holds for any $R \geq 1$ : we cover $S(0, R)$ with $\tilde{v}_{n}\left(T, \frac{1}{2}\right)$ balls of radius $\frac{1}{2}$ in a way that each ball intersects $S(0, R)$ in an $(n-2)$-sphere of radius $\frac{1}{2}$. Then the balls cover the annulus between $S(0, R)$ and $S(0, R-1 / 2 R)$. Writing $m$ to denote the maximal integer such that $T-m / 2 T \geq \frac{1}{2}$, it follows by (3.15) that

$$
\begin{aligned}
\nu_{T, n} & \leq 1+\sum_{i=0}^{m} \tilde{v}_{n}\left(T-\frac{i}{2 T}, \frac{1}{2}\right) \\
& \ll 2^{n-1} n \sqrt{n} \ln n \sum_{i=0}^{m}\left(T-\frac{i}{2 T}\right)^{n-1} \\
& <2^{n-1} n \sqrt{n} \ln n \cdot \frac{T^{n-1}}{1-e^{-(n-1) / 2 T^{2}}} \\
& \ll \frac{n \sqrt{n} \ln n}{T} \cdot(2 T)^{n},
\end{aligned}
$$

using $(n-1) / 2 T^{2}>1$ in the last step.

If $\frac{1}{2}+1 / 4 n \leq T \leq 1$, then it is essentially sufficient to cover $S(0, T)$, hence (3.16) yields (3.8).

Finally, if $\frac{1}{2}<T \leq \frac{1}{2}+1 / 4 n$, then the balls centred at the vertices of the inscribed regular crosspolytope show (3.9) [BW].

\subsection{About the Lower Bounds}

The lower bound (3.10) for $T \geq n / 2$ follows essentially directly from the celebrated lower bound of order $n$ on the covering density of a ball, which bound is due to Coxeter 
et al. [CFR]. In addition, (3.14) is a consequence of (3.17) because the balls covering $B(0, T)$ cover $S(0, T)$ as well. Now (3.10) for $T=\sqrt{n} / 2$ is a consequence of (3.14).

If conjectures (3.18) and (3.19) hold, then they yield (3.11) and (3.12).

\section{Acknowledgments}

The author thanks Roland Bacher and Gilbert Muraz for very helpful suggestions and the anonymous referee for very useful comments concerning the asymptotic estimates.

\section{References}

[BL] J. Bourgain and J. Lindenstrauss, On covering a set in $\mathbb{R}^{n}$ by balls of the same diameter, in J. Lindenstrauss and V.D. Milman (eds.), Geometric Aspects of Functional Analysis (1989-90), Lecture Notes in Mathematics, vol. 1469, Springer-Verlag, Berlin, 1991, pp. 138-144.

[BW] K. Böröczky, Jr. and G. Wintsche, Covering the sphere by equal spherical balls, in Discrete and Computational Geometry, The Goodman-Pollack Festschrift, Algorithms and Combinatorics, vol. 25, Springer-Verlag, Berlin, 2003, pp. 237-253.

[CFR] H.S.M. Coxeter, L. Few and C.A. Rogers, Covering space with equal spheres, Mathematika 6 (1959), $147-157$.

[F] G. Fejes-Toth, Multiple packing and covering of spheres, Acta Math. Acad. Sci. Hungar. 34 (1979), $165-176$.

[FF] P. Frankl and Z. Furedi, Solution of the Littlewood-Offord problem in high dimensions, Ann. of Math. 128 (1988), 259-270.

[GO] J.E. Goodman and J.O'Rourke (editors), Handbook of Discrete and Computational Geometry, CRC Press, Boca Raton, FL, 1997.

[IM] V.D. Ivashchuk and V.N. Melnikov, Billiard representation for multidimensional cosmology with intersecting $p$-branes near the singularity, J. Math. Phys. 41 (2000), 6341-6363.

[K] R. Kerschner, The number of circles covering a set, Amer. J. Math. 61 (1939), 665-671.

[M] P. Matthews, Covering problems for Brownian-motion on spheres, Ann. Probab. 16 (1988), 189-199.

[MR] J. Matoušek and Y. Rabinovich, On dominated $l_{1}$ metrics, Israel J. Math. 123 (2001), 285-301.

[MVG] G. Muraz and J.-L. Verger-Gaugry, On lower bounds of the density of packings of equal spheres of $\mathbb{R}^{n}$, Preprint 580, Institut Fourier, 2003.

[R1] A.M. Raigorodskii, Borsuk's problem and the chromatic numbers of some metric spaces, Russian Math. Surveys 56 (2001), 107-146.

[R2] C. A. Rogers, Covering a sphere with spheres, Mathematika 10 (1963), 157-164.

[R3] C. A. Rogers, Packing and Covering, Cambridge University Press, Cambridge, 1964.

[S] O. Schramm, Illuminating sets of constant width, Mathematika 35 (1988), 180-189.

[V] G. Valiron, Théorie des fonctions, Masson, Paris, 1966.

Received December 13, 2002, and in revised form July 1, 2003. Online publication June 4, 2004. 